\title{
Evaluation of the Myocardial Motion at Scar Locations Using 4D MDCT Cardiac Images
}

\author{
Wei-Chih $\mathrm{Hu}^{1}$, Hsuan-Ming Tsao ${ }^{2}$ \\ ${ }^{1}$ Chung Yuan Christian University, Chung Li, Taoyuan, Taiwan \\ ${ }^{2}$ National Yang Ming University, Taipei, Taiwan
}

\begin{abstract}
This study sought to characterize global and regional systolic function abnormalities. The self-developed $4 D$ image processing software will be extracting the wall motion of left ventricle from a $4 D$ cardiac images. A seeded region growing incorporated active contour method was designed to locate the edge of endocardial and epicardium. A $3 D$ triangle mesh was utilized to construct a $3 D$ grid model of heart. The magnitude of myocardial wall motion in different extent will be marked with colors in $3 D$ model. The coordinates of lower motion areas and related $L V$ parameters were characterized and recorded. Four data set of fibrotic scar delay enhanced cardiac images and $4 D$ cardiac wall motion images was used to test this non-invasive method of early detection cardiac abnormality. The patient of ventricular fibrillation after radio ablation with fibrotic scar tissue necessarily has lower myocardial motion value. However, the fibrotic scar tissue will not reveal signature of delay enhances up to $40 \%$ of low motion areas but the operated area may still have lower myocardial motion.
\end{abstract}

\section{Introduction}

Cardiac disease is the challenging clinical problem. It is often responsible to regional myocardial injury. The synergy of myocardial contraction creates an effective cardiovascular pumping system. Regional dysfunction of myocardium will be leading to functional abnormality of cardiac wall motion that is one of the risk factors for ventricular remodelling and heart failure. Early detecting abnormality of wall motion and treated could prevent further deterioration.

To early evaluation of cardiac functions, the cardiac imaging and image analysis methods to measure the regional function of the left ventricle $(\mathrm{LV})$, through $\mathrm{LV}$ wall motion and strain measurement and infarcted myocardium may be the best solution. There are many existing techniques which have been unnavigable in making more precise and reliable quantitative regional $\mathrm{LV}$ function because of the motion of heart is a non-rigid that rotates and deforms in 3D space. The common evaluation of heart is mostly using $2 \mathrm{D}$ image sequence data [1-6]. Moreover, most of these techniques is only using the end-diastolic (ED) and end systolic (ES) image frames, while the LV actually pass through a temporal wave of contraction, wall thickening and relaxation $[2,4$, 6].

The invasive technique cannot be used clinically on the study of human heart. A recent technique is the use of phase contrast MR images to decipher local velocity. It can be integrated to estimate trajectories of individual points over time. Song and Leahy have developed fluid flow models with the optical flow approach using 3D dataset [7]. Kambhamettu and Goldgof utilized shape matching idea with Gaussian curvature and conformal stretching models to simulate non-rigid motion[8]. There are studies using modal finite element analysis to study non-rigid motion $[9,10]$ that led to Monga and Ayache group discussed the segmentation and tracking the object simultaneously [11].

We hypothesize that the shape of the endocardial and epicardial surfaces could lead us to find the 4D trajectories of central axis of left ventricular, at any given time instant, over the entire cardiac cycle, based on given instant of ventricular geometric centre. It will offer the advantages for quantifying myocardial function noninvasively such as wall motion. In this paper, we use scar tissue to correlate with low motion area of left ventricular of human hearts that identify the injury area of myocardium. Therefore, this study seeks to develop a 4D image processing software will be extracting the wall motion of left ventricle from a 4D cardiac images that could be evaluating the wall motion to indicate the dysfunction area of myocardium.

\section{Method and Material}

The study of wall motion in relationship to Radiofrequency Ablation scar tissue of left ventricular using 4D Cardiac image has been grouped into following:

\subsection{Data Extraction}


The 4D self-developed image processing software has been developed with Microsoft Windows 7 on a 64 bit platform with Intel Celeron CPU G550, memory DDR3 4GB, Visual $\mathrm{C}++6.0 \mathrm{MFC} 4.2$ and OpenGL library. 4D Cardiac images are acquired at the National Institute of Hospital of Yang Ming, National Yang Ming University using by the Philips computerized tomography. The study was approved by the Institutional Review Board of National Yang Ming University. The informed consent procedure has been performed to each subject/patient. Each subject has been acquired 10 sets of timing frames of 3D cardiac volume that cover a complete heartbeat cycle that stored in DICOM (Digital Imaging and Communications in Medicine) format. The scanned image size is $512 \times 512$ (pixels).

The self-developed image processing program will read the complete $4 \mathrm{D}$ images into the program. 3D reorganization and image information is obtained to construct the stereoscopic model of the thoracic area. First, select the left ventricular cut angle for all other timing use. The program will be using the select view to search for the left ventricular central axis and resample the 4D data set into the long axis view. The split page of heart structure manually is illustrated as Figure 1. The regional growth algorithm will delineate the contour of the left ventricular endocardium. Repeated segmentation is performed to obtain epicardial information. The 3D model of left ventricular endocardium (Figure 2) and epicardium is established by triangular mesh shown as Figure 3. The endocardial and epicardial information is subtracted to obtain left ventricular myocardial information. After the construction of ten time models, the model of left ventricular endocardium, epicardial movement and the model of myocardial are observed by program.
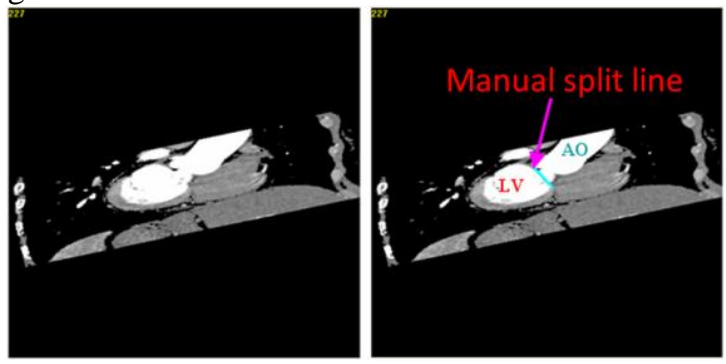

Fig1.Manual separated line that separates the left ventricle from the aorta
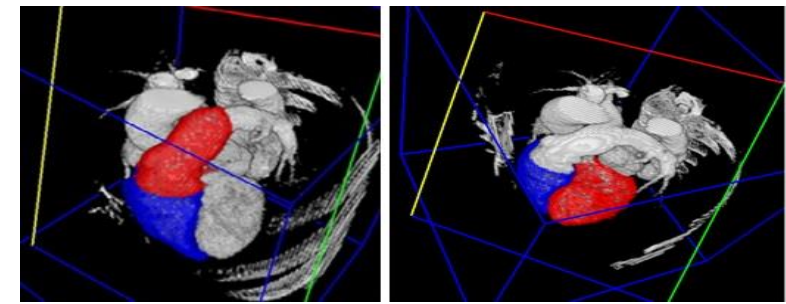

Figure 2. 3D illustration of heart. At left, blue colour is showing right ventricle and red colour is show pulmonary artery. At right, the red colour is showing left ventricle.



Fig.3 Left Ventricular Simulated Model. At left, the triangular mesh the green colour is for epicardial model, the red colour is for the endometrial model. The right panel shows the model of wall motion.

\subsection{Fibrosis area assessment interface}

The study explores the relationship between postoperative region and cardiac motion. The delayedenhancement images are used to determine area of fibrotic tissue. The average and standard deviation of $\mathrm{HU}$ (Hounsfield unit) will be calculated with a single click of the right mouse button at the 10 points radius to the centre of mouse click point as shown in Figure 4. We get the region of interest $\mathrm{HU}$ value which shows this region of fibrosis. This experiment was performed to find the fibrosis area.

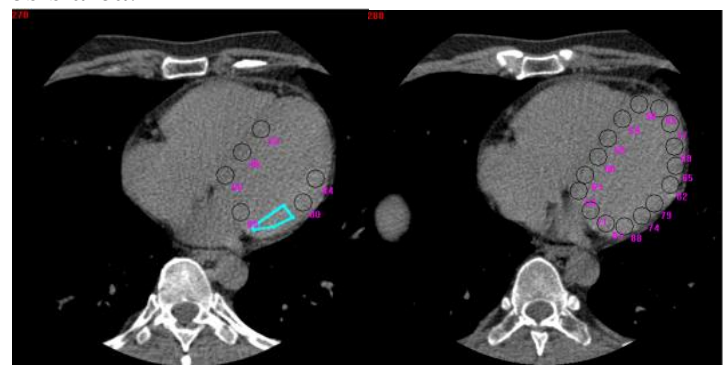

Fig.4 Fibrous area assessment interface (Pink circle area is the $\mathrm{HU}$ value of the manual selection area)

\section{Result and discussion}

The result section will present the simulation system that exhibits the left ventricular motion and the delayed enhancement pixel calculation. Then, we will illustrate the relationship of the compared of data.

\subsection{Ventricular wall motion}

3D heart volume of 10 lattice grid models from timing 0 to 9 was reconstructed into consecutive data set. This is a four-dimensional model of left ventricular in motion. This display interface allows the user to observe the three-dimensional model of the inner portion of the left ventricle (Fig 5).

The left ventricular model change motion, different colour areas represent the difference between the mean left ventricular displacements. The blue is the relative displacement of $2 \mathrm{~mm}$ or less, the light blue is the 
displacement $2 \sim 3 \mathrm{~mm}$, green is the displacement of 3 $\sim 4 \mathrm{~mm}$, yellow is the displacement of $4 \sim 5 \mathrm{~mm}$, red is the displacement of more than $5 \mathrm{~mm}$, and low-motion area is defined as the average amount of difference between the value of less than $2 \mathrm{~mm}$ area,

This study re-sampled after the left ventricular model 1 to 10 layers defined as the base layer, 11 to 20 layers is defined as the middle layer, 21 to 30 layers defined as the top layer. The method takes 30 points of sampling points counter clockwise.

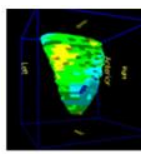

$0 \%$

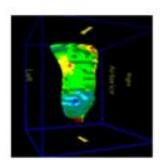

$50 \%$
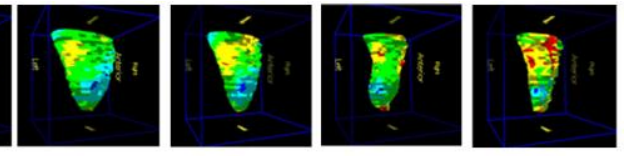

$10 \%$

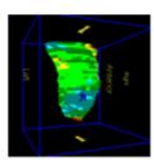

$20 \%$



$60 \% \quad \begin{gathered}70 \% \\ \text { Result of wall motion }\end{gathered}$

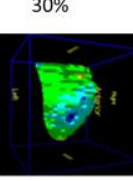

$80 \%$

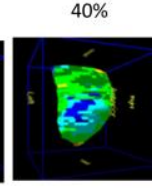

$90 \%$

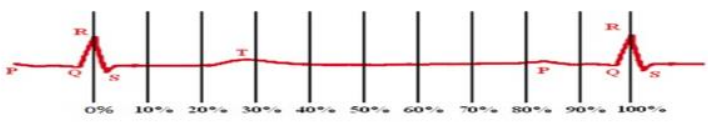

Figure 6. The illustration of Wall motion in graphical display. The blue colour represents the area of motion less than $2 \mathrm{~mm}$

\subsection{Statistics of wall motion}

We observed the low-motion region that occurs in the left ventricle and defined 1 to 15 points for the front side (inferior), 16 to 30 points for the back (inferior), each interval is 12 degrees sampling points (Figure 6). In addition, the movement model analysis interface can be calculated with low sequence and the radius change rate (Table.1).
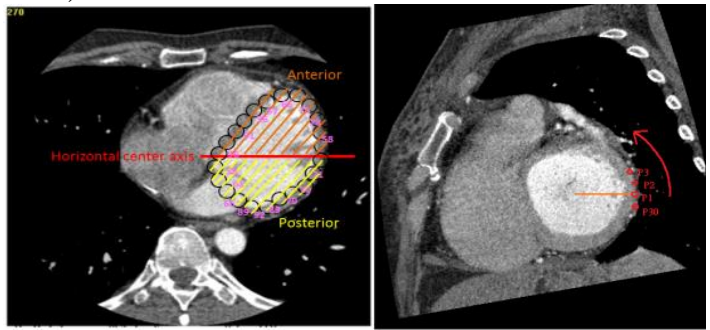

Figure 6. The definition of position and origination about the image data.

\subsection{Statistics of scar area}

It is known as the surgical area in the first few slices , a point group is greater than $77 \mathrm{HU}$ values that can be detected on the slice as described before, i.e., a point group is greater than 2 standard deviations of the average $\mathrm{HU}$ value. It is found that the region with fibrosis is associated with the surrounding area. The low-volume region is the fibrotic area. Therefore, according to the results of the graphs, the following results are found.

We have four Subjects (Patients), Subject 1and 4 are matching low motion of surgical area with scar tissue area. Patient 2 has five districts surgical area, presence of fibrous tissue is only two regions (about 40\%), compared with the 2SD (Standard Deviation) region. The region is higher than the average of 1.6 to 1.8 times the SD value produced.

Table 1. The illustration of wall motion

\begin{tabular}{|l|l|l|l|}
\hline layer & Segment group location & Motion & Location \\
\hline 8 & segment 23, Degree 276 & $1.712 \mathrm{~mm}$ & Middle \\
\cline { 2 - 3 } & Segments 24,Degree 288 & $1.784 \mathrm{~mm}$ & layer \\
\multirow{3}{*}{9} & Rear side \\
\cline { 2 - 3 } & Segments 23, Degree 276 & $1.672 \mathrm{~mm}$ & \\
& Segments 24, 288 degree & $1.559 \mathrm{~mm}$ & \\
& Segments 25, 300 degree & $1.588 \mathrm{~mm}$ \\
\cline { 2 - 3 } & Segments 26, 312 degree & $1.576 \mathrm{~mm}$ \\
\hline 10 & Segments 23, 276 degree & $1.695 \mathrm{~mm}$ \\
\cline { 2 - 3 } & Segments 24, 288 degree & $1.701 \mathrm{~mm}$ \\
\cline { 2 - 3 } & segments 25, 300 degree & $1.543 \mathrm{~mm}$ \\
\hline 11 & Segments 24, 288 degree & $1.728 \mathrm{~mm}$ & \\
\hline
\end{tabular}

\subsection{Discussion of the Comparison of wall motion and scar area}

Compared with larger than 2SD region, low-motion region found, the fibrotic area completely overlapped with low-motion region which indicate the fibrotic area, has a significant correlation with low-motion region. As shown in Table 1, Subject 1, middle layer, posterior side, CT image slice from 268 to 270 , as illustrated, there are many location that matching the low wall motion area. This study is also found that the fibrosis area is significantly lower than low exercise area, injured area involved in the movement of adjacent muscles which led to low mobility area than the fibrosis area to the wide, Subject 1, middle layer, posterior side, there are no fibrotic tissue found in CT image (image data 266 and 267 as illustrated in Table 1) in this region, as long as the myocardial injury to a certain extent will lead to low motion incidence of the region, but the system can still find the low motion region and position. Only the lowmotion area is the medium-risk group in the non-scar area This study is compared with the fibrotic area method which is proposed by the previous research, our study involves to detect the low motion area. It is possible to predict the location of the fibrosis region by irradiating the delayed-enhancement image, the tool can further identify the area of low motion activity without fibrosis, which is performed by the physician for early monitoring, and tracking can provide effective help. 
Table 2. The illustration of comparison

The HU pixel value in relationship to wall motion

\begin{tabular}{|c|c|c|c|c|}
\hline $\begin{array}{l}\text { The } \\
\text { slice of } \\
\text { Image Data }\end{array}$ & $\begin{array}{l}\mathrm{HU} \text { value is greater than } \\
\text { the average 2SD } \\
\text { area(HU value }>77)\end{array}$ & layer & location & Motion \\
\hline 266 & No & \multirow[t]{5}{*}{$11^{\mathrm{th}}$} & $276^{\circ}$ & $1.712 \mathrm{~mm}$ \\
\hline 267 & No & & \multirow[t]{4}{*}{$288^{\circ}$} & \multirow[t]{4}{*}{$1.784 \mathrm{~mm}$} \\
\hline 268 & $\begin{array}{ll}264^{\circ} & \mathrm{HU}=85 \\
276^{\circ} & \mathrm{HU}=77 \\
\end{array}$ & & & \\
\hline 269 & $\begin{array}{ll}264^{\circ} & \mathrm{HU}=86 \\
276^{\circ} & \mathrm{HU}=92 \\
336^{\circ} & \mathrm{HU}=79 \\
348^{\circ} & \mathrm{HU}=77 \\
\end{array}$ & & & \\
\hline 270 & $\begin{array}{ll}264^{\circ} & \mathrm{HU}=89 \\
276^{\circ} & \mathrm{HU}=92 \\
288^{\circ} & \mathrm{HU}=83 \\
312^{\circ} & \mathrm{HU}=79 \\
336^{\circ} & \mathrm{HU}=79\end{array}$ & & & \\
\hline
\end{tabular}

\section{Conclusion}

This research is evaluated low motion of the left ventricle after surgery. It is to compare the motion of myocardium changes and fibrosis of the radio ablation region. Furthermore, it is to confirm that it is feasible to evaluate the dysfunction of myocardium through the analysis of low wall motion. We can apply this method to different types of ventricle disease of the cardiomyopathy, this study is included the assessment of myocardial infarction, Cardiac remodelling and assessing the role of fibrotic tissue in the heart. Further research will involve integration of the heart motion, validating geometrical landmarks and integration of the motion matching. This method is sensitive to detect the myocardium dysfunction. However, more data should be continuously gathering.

\section{Acknowledgements}

The research was support by grant from Ministry of Science and Technology, MOST- 104-2221-E-033 -067 -.

\section{References}

[1] Areeda J, Garcia E, Vantrain K, Brown D, Waxman A. Berman D. A comprehensive method for automatic analysis of rest/exercise ventricular function from radionuclide angiography. Digital Imaging: Clinical Advances in Nuclear Medicine, 1982, 241-256.

[2] Bolson EL, Kliman S, Sheehan F, Harold TD. Left ventricular segmental wall motion - A new method using local direction information. Computer in Cardiology, 1980, 245-248.

Clayton PD, Bulawa WF, Klausner SC, Urie PM, Marshall HW, Warner HR. The characteristic sequence for the onset of contraction in the normal left ventricle. Circulation, 1979, 59:671-679.

Gelberg HJ, Brundage BH, Glantz S, Parmley WW. Quantitative left ventricular wall motion analysis: A comparison of area, chord and radial methods. Circulation, 1979, 59:991-1000.

[5] Slager CJ, Hooghoudt THE, Serruys PW, Schuurbiers JCH, Reiber JHC, Meester GT, Verdouw PD, Hugenholtz PG. Quantitative assessment of regional left ventricular motion using endocardial landmarks. JACC, 1986, 7( 2):3 17-326.

[6] Zissermann D, Strand EM, Smith LR, Wixson SE. Hood WPJr, Mantle JA, Rogers WJ, , Rackley CE. Cardiac catheterization and angiographic analysis computer applications. Progress in Cardiovascular Diseases. 1983, 25 (5):409-434.

[7] Song SM, Leahy RM. Computation of 3D velocity fields from 3D cine CT images. IEEE Transactions on Medical Imaging. 1991, 10 (3):295-306.

[8] Kambhamettu C, Goldgof DB. Point correspondence recovery in non-rigid motion. IEEE Computer Vision and Pattern Recognition, 1992, 222- 227.

[9] Pentland A, Horowitz B. Recovery of non-rigid motion and structure. IEEE Transactions on Pattern Analysis and Machine Intelligence, 1991, 13 (7), 730-742.

[10] Terzopoulos D, Metaxas D. Dynamic 3D models with local and global deformations: deformable super quadrics. IEEE Transactions on Pattern Analysis and Machine Intelligence, 1990, 13(7), 606-615.

[11] Monga O, Ayache N, Sander P. From voxel to curvature. IEEE Computer Vision and Pattern Recognition, 1991, 644-649.

[12] Sander PT, Zucker SW. Inferring surface trace and differential structure from 3D images. IEEE Transactions on Pattern Analysis and Machine Intelligence, 1990, 12(9):833-854.

[13] Park J, Metaxas D, Young AA, Axel L. Deformable models with parameter functions for cardiac motion analysis from tagged MRI data. IEEE Transactions on Medical Imaging, 1996, 15(3), 278-289.

[14] Huang J, Abendschein D. Davila-Roman VG, and Amini AA. Spatio-temporal tracking of myocardial deformations with a 4-D B-spline model from tagged MRI. IEEE Transactions on Medical Imaging. 1999, 18 (10), 957-972.

[15] Smith MF. The effect of contraction and twist on myocardial PET and SPECT image resolution: a mathematical phantom study. IEEE Transactions on nuclear science, 2000. 47 (4), 16461654.

[16] Staib LH, Duncan JS. Deformable Fourier models for surface finding in 3D images. SPIE Vol. 1808: Visualization in Biomedical Computing, 1992, 90-104.

[17] Razi T, Niknami M, Ghazani FA. Relationship between hounsfield unit in CT scan and gray scale in CBCT. Journal of dental research, dental clinics, dental prospects, 2014, 8(2), 107110 .

Address for correspondence.

Weichih $\mathrm{Hu}$.

Dept. of Biomedical Engineering

Chung Yuan Christian University

\#200, Chung Pei Rd., Chung Li, Taoyuan

Taiwan, 32023.

weichihhu@cycu.edu.tw 